\title{
РАЗРАБОТКА КОНЦЕПТУАЛЬНОЙ МОДЕЛИ РЕСТРУКТУРИЗАЦИИ ОРГАНИЗАЦИОННОЙ СТРУКТУРЫ УПРАВЛЕНИЯ СПЕЦАВТОХОЗЯЙСТВ
}

\author{
Григорьева Татьяна Евгеньевна 1 , \\ tanya_grig_1991@mail.ru \\ Дегтярева Наталия Алексеевна², \\ zxasqw@scalpnet.ru
}

\begin{abstract}
1 Томский государственный университет систем управления и радиоэлектроники, Россия, 634050, г. Томск, пр. Ленина, 40.

2 Томский филиал Российской академии народного хозяйства и государственной службы при Президенте Российской Федерации, Россия, 634050, г. Томск, пр. Ленина, 99.
\end{abstract}

Григорьева Татьяна Евгеньевна, аспирант кафедры компьютерных систем в управлении и проектировании Томского государственного университета систем управления и радиоэлектроники.

Дегтярева Наталия Алексеевна, кандидат исторических наук, заведующая кафедрой государственного и муниципального управления Томского филиала Российской академии народного хозяйства и государственной службы при Президенте Российской Федерации.

В поддержании благосостояния города, его функционирования и жизнеобеспечения задействованы различные учреждения, например ЖКХ, управляющие компании, УМП «Спецавтохозяйство» и другие организации, которые создают населению комфортную городскую среду. Особую роль в поддержании этой среды играет деятельность спецавтохозяйств, работа которых приобретает значимость в период выпадения обильных осадков в виде снега, что сопровождается затруднительным передвижением транспорта. Также несвоевременно убранный снег негативно сказывается на экологическом состоянии города. Анализ обстановки на дорогах показывает, что процессы зимнего содержания дорог требуют особой оперативности в их организации и управлении. Объектом исследования является обобщенная организационная структура управления спещавтохозяйств, которая соответствует линейно-функциональному типу. При такой структуре имеется ряд недостатков, которые впоследствии приводят к потере оперативности принятия решений, что так важно в деятельности спецавтохозяйств, т. к. они имеют тесную взаимосвязь с динамически изменяющейся окружающей средой. В связи с этим целью статьи является разработка концептуальной модели реструктуризации существующей структуры управления спещавтохозяйств. При разработке такой модели использовались методы теоретического анализа с элементами проектирования. Предложенная модель представляет собой функциональную организационную структуру с элементами линейной и позволит спецавтохозяйствам достичь оперативности принятия управленческих решений, избежать дублирования функций между отделами и улучшить коммуникацию между ними. При переходе к предлагаемой структуре преимущества существующей сохраняются, а недостатки минимизируются. Более того, предлагаемая модель является динамичной и гибкой при переходе к другому типу структур: например, в случае участия спецавтохозяйств в национальных, федеральных проектах, региональных, муниципальных и других программах в организационную структуру необходимо добавить проектно-матричные элементы.

Ключевые слова: Зимнее содержание дорог, спецавтохозяйства, принятие управленческих решений, организационная структура и ее реструктуризация, городская среда. 


\section{Введение}

Комфортная городская среда формируется из совокупности соответствующего обслуживающего комплекса, который функционирует и развивается преимущественно для удовлетворения потребностей жителей. В развитии города можно выделить несколько организаций и учреждений, которые взаимно обеспечивают функционирование друг друга и при этом город взаимодействует не только с ними, но и с внешней средой.

В настоящее время данная тематика приобретает особую актуальность по причине того, что в 2019-2024 гг. наблюдается развитие национальных проектов России, а именно комфортной городской среды для жизни населения [1].

Для поддержания комфортной городской среды следует обратить внимание на содержание и уборку городских улиц, что является важным фактором нормального функционирования города и жизнеобеспечения его населения, например на удовлетворительное состояние автомобильных дорог, отсутствие «пробок» на них, благоприятные экологические условия и т. д.

Так, период выпадения обильных осадков в виде снега сопровождается затруднительным передвижением транспорта, появляются «пробки», заторы, что является серьезной проблемой на узких дорогах городов $[2,3]$.

Учитывая экологическую составляющую, снег является фильтром-накопителем химических и других загрязняющих веществ, выбрасываемых в атмосферу автомобилями, заводскими и городскими теплоэнергетическими установками, работающими на углеводородном топливе, что негативно сказывается на экологическом состоянии городов [4-6].

Анализ обстановки на дорогах показывает, что зимнее содержание дорог является трудоёмкой работой, требующей особой оперативности в управлении внутренними процессами с учетом рационального использования ресурсов [7].

Исходя из условий поддержания жизнеобеспечения городского населения актуализируется работа по координации и организации зимней уборки автомобильных дорог, осуществляющаяся спецавтохозяйствами. В соответствии с «Методическими рекомендациями по зимнему содержанию автомобильных дорог с использованием специализированной гидрометеорологической информации (для опытного применения)» основной их задачей является качественное и оперативное выполнение комплекса дорожных работ по созданию необходимых условий бесперебойного и безопасного движения автомобильного транспорта по дороге и искусственных сооружений на них [8].

Особую роль в процессах зимнего содержания дорог играют погодноклиматические условия, которые относятся к «неуправляемым факторам», но в то же время системе управления спецавтохозяйств следует оперативно на них реагировать. Как показывает практика, спецавтохозяйства к обильным снегопадам порой оказываются не готовы, что является следствием проблемы оперативности в процессе принятия управленческих решений (ПУР) [9]. Среди причин указанной проблемы можно выделить наиболее значимую - взаимодействие элементов организационной структуры спецавтохозяйств.

Таким образом, целью статьи является разработка концептуальной модели реструктуризации организационной структуры управления спецавтохозяйств, которая позволит более оперативно реагировать на изменяющиеся внешние условия.

Анализируя исследования в данной области, можно сделать вывод, что большинство работ направлены на повышение эффективности управления жилищнокоммунальным хозяйством на муниципальном уровне [10] и на уровне социальноэкономического развития города [11-14]. Однако анализ организационной структуры 
спецавтохозяйств и её влияние на эффективность реагирования всей системы управления в работах исследователей не прослеживаются.

\section{Процесс разработки и принятия управленческих решений в деятельности спецавтохозяйств}

Современные условия процесса ПУР требуют от руководителей спецавтохозяйств разработки обоснованных и эффективных решений, которые дают оптимальный результат.

Последствия неправильных и нерациональных управленческих решений значительны, т. к. от принимаемого решения руководителя зависит результативность всей системы управления [15]. Например, если на этапе погрузки снега в самосвалы выделено предостаточное количество техники, то часть ее может проработать впустую, затратив только финансовые средства на бензин.

Модель принятия управленческих решений руководителями спецавтохозяйств вписывается в российскую школу управления, согласно которой решения ими принимаются на каждом уровне управления: постановка проблемы, разработка вариантов решения, выбор наилучшего варианта. В процессе ПУР такой модели в основном используются качественные (интуитивные) методы. Но поскольку они являются наиболее рискованными, необходимо применять и количественные методы.

Так, в работе [16] авторами статьи предложен комплексный подход к выбору методов, применяемых при разработке управленческих решений, позволяющий сочетать использование количественных и качественных методов. К тому же на своевременность разработки и принятия управленческих решений влияют: количество и профессионализм кадрового состава, объем и ценность располагаемой информации, интенсивность связей между подразделениями внутри предприятия и т. п.

Исходя из вышесказанного, в данной статье предлагается рассмотреть организационную структуру управления спецавтохозяйств, выявить «узкие места» существующей структуры и предложить модель ее реструктуризации, которая повлияет на оперативность принятия управленческих решений. На основе предложенной модели структуры сформированы показатели организационной эффективности, позволяющие определить степень оперативности принимаемых решений.

\section{Обобщенная организационная структура управления спецавтохозяйств}

Среди типов организационных структур, наибольшую популярность имеет линейно-функциональная структура, которая позволяет в значительной степени устранить недостатки как линейного, так и функционального управления.

Анализируя организационные структуры спецавтохозяйств различных городов $[17,18]$, было выявлено, что они соответствуют линейно-функциональному типу, но отличаются друг от друга количеством и наименованием отделов.

На рис. 1 представлена обобщенная организационная структура управления спецавтохозяйств.

В такой структуре у директора появляются линейные руководители, функции которых соответствуют целям их подразделений. Для данной структуры характерен вертикальный процесс ПУР, т. е. сверху вниз.

Линейно-функциональная структура имеет ряд преимуществ и недостатков. Среди преимуществ выделяют:

- рациональное сочетание линейных и функциональных взаимосвязей;

- стабильность полномочий и ответственности за сотрудников; 
- единство и четкость распорядительства;

- личная ответственность каждого руководителя за результаты деятельности;

- профессиональное решение задач специалистами функциональных служб.

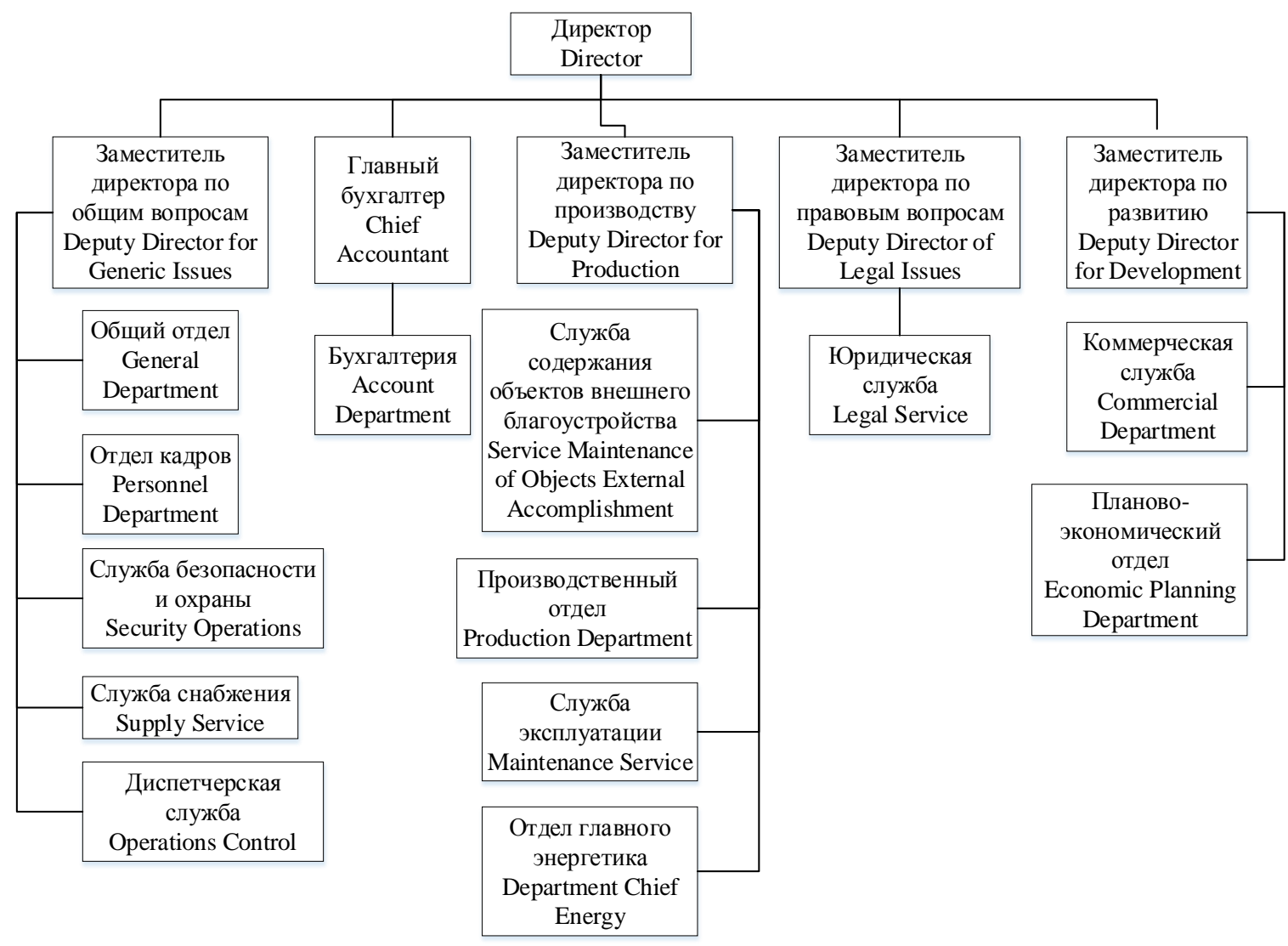

Pис. 1. Обобщенная организационная структура управления спецавтохозяйств Fig. 1. Generalized organizational structure of special motor transport service management

К недостаткам можно отнести дублирование функций руководителя и функциональных специалистов, из-за чего теряется время на выполнение одной и той же задачи. Так, например, анализируя организационную структуру УМП «Спецавтохозяйство г. Томска», выявлено, что в управлении у заместителя директора по развитию имеются планово-экономический и финансовый отделы, функции которых перекликаются в рамках внутреннего финансового контроля соблюдения финансовой дисциплины при заключении договоров и осуществлении экономической, хозяйственной деятельности предприятия. Исходя из дублирования функций, теряется оперативность ПУР, т. к. решения принимаются через посредника. Разногласия между линейными и функциональными службами повышают риск неправильного толкования информации, передаваемой линейным исполнителям функциональными руководителями, что сопровождается многоярусной коммуникацией.

Исходя из недостатков и особенностей линейно-функциональной организационной структуры, возникают сложности при расчете трудоемкости работ и нормы управляемости, что сказывается в дальнейшем на определении ее эффективности. 


\section{Концептуальная модель реструктуризации организационной структуры управления спецавтохозяйств}

Руководителям предприятий периодически необходимо оценивать и корректировать поставленные цели с учетом изменений внешней среды и внутренних процессов предприятия. Развитие предприятий спецавтохозяйств может осуществляться за счет изменений в технологическом процессе уборки улиц, использования современной снегоуборочной техники, модернизации системы стимулирования и пр., что носит фрагментарный характер и может не дать полноценного эффекта. Для того чтобы преобразования касались не только объекта и инструментов управления, но и субъекта, необходимо учитывать и усовершенствовать базисный элемент (т. е. организационную структуру предприятия), который является объединяющим, интегрирующим и обеспечивающим гибкость процесса коммуникации между структурными элементами системы.

На рис. 2 представлена предлагаемая концептуальная модель реструктуризации организационной структуры управления спецавтохозяйств.

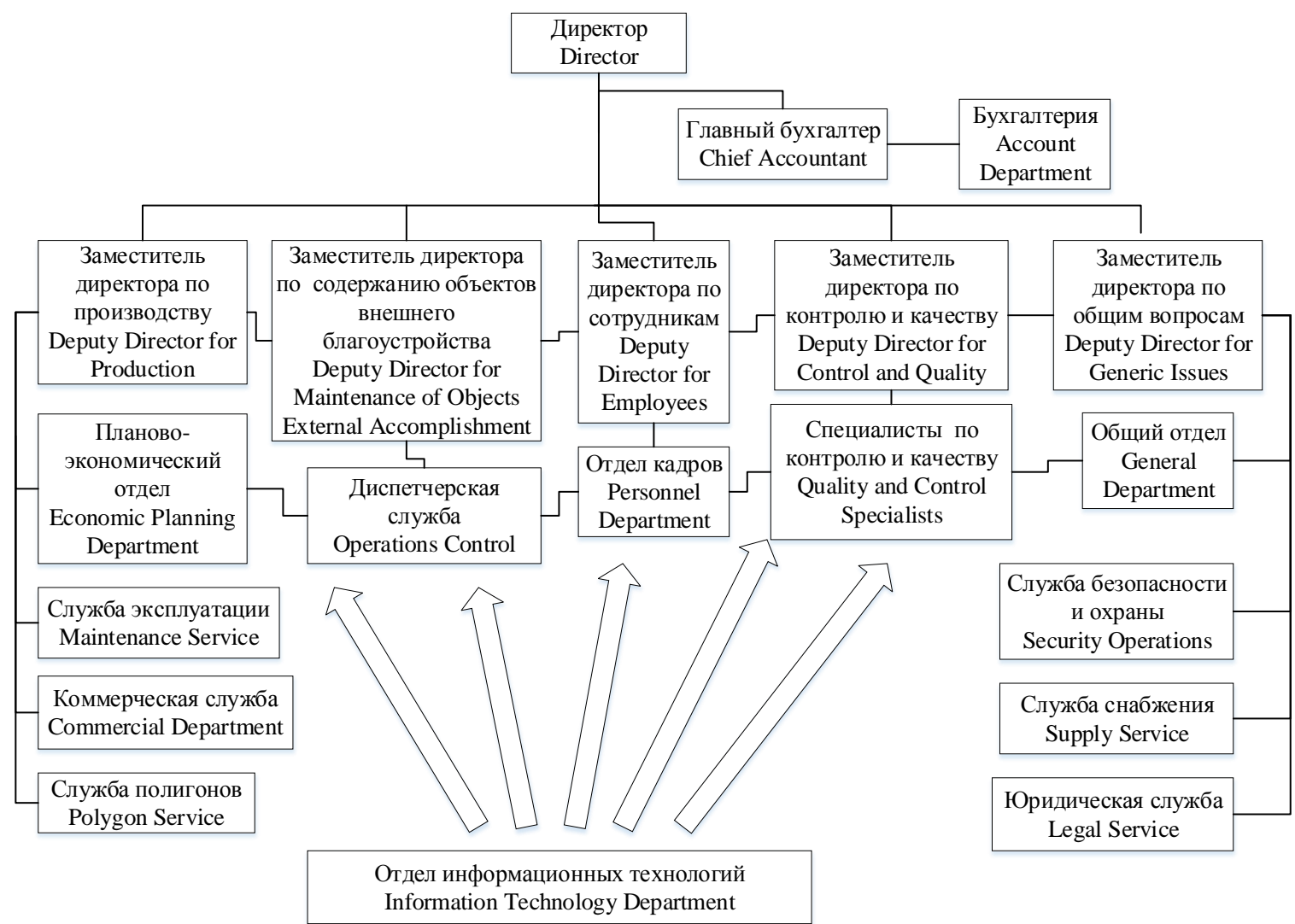

Puс. 2. Концептуальная модель реструктуризации организационной структуры управления спецавтохозяйств

Fig. 2. Conceptual model of restructuring the organizational structure of special motor transport service management

Предлагаемая модель системы управления соответствует функциональной организационной структуре с элементами линейной.

Центральным звеном сверху является директор, у которого в подчинении заместители каждого отдела и главный бухгалтер. Отделы сформированы по функциям управления, т. е. заместитель директора по производству осуществляет функции пла- 
нирования (например, разработка технологических карт, согласно которым производится уборка улиц и т. д.), заместитель директора по содержанию объектов внешнего благоустройства - функции организации (но с учетом планирования того, что будет организовано), заместитель директора по сотрудникам - функции мотивации, заместитель директора по контролю и качеству - функции контроля, заместитель директора по общим вопросам - состоит из всеобщих отделов, которые имеют линейные связи со всеми другими отделами. Каждый отдел, в свою очередь, состоит из мелких вспомогательных отделов. Центральным звеном снизу является отдел информационных технологий, который будет отвечать за обеспечение и использование современными технологиями, инновациями не только общих отделов (например, обеспечение современными компьютерами, программными продуктами и т. д.), но и специализации предприятия (например, обеспечение современной снегоуборочной техникой, применение новых технологий уборки и т. д.), а также предлагать современные инструменты процесса ПУР (например, при планировании процесса снегоуборки применить имитационное моделирование).

В отличие от линейно-функциональной структуры функциональная организационная структура имеет следующие преимущества:

- более глубокая проработка решений по функциональным направлениям, т. е. более детальное планирование процессов своей специализации;

- высокая компетентность специалистов, отвечающих за выполнение функций, что сопровождается систематическим участием сотрудников в реализации региональных, муниципальных и других программах, например участие в проекте благоустройства общественных пространств [19];

- высвобождение линейных руководителей от части задач, решаемых функциональными, что позволит избежать дублирование выполняемых функций. Исходя из приведенного примера, при переходе от линейно-функциональной организационной структуры к предложенной у планово-экономического и финансового отделов будут разграничены выполняемые функции.

Очевидно, что идеальной модели организационной структуры не существует, поэтому имеются риски следующих недостатков:

- ориентация на реализацию в основном закрепленных функций, т. е. узость и отсутствие более широкого вида перспектив, горизонта;

- отсутствие инновационности и гибкости при изменении ситуации как в организации, так и во вне ее - для устранения этого недостатка в организационной структуре центральным звеном снизу предлагается сделать отдел информационных технологий и добавить линейные связи между отделами.

Сопоставив линейно-функциональную и функциональную организационную структуры, можно сделать вывод, что при переходе от линейно-функциональной к функциональной организационной структуре преимущества линейно-функциональной структуры сохраняются, а недостатки ее минимизируются.

На основе предложенной модели структуры формируются следующие показатели организационной эффективности:

- уровень достижения поставленной цели, который формируется за счет количества подразделений и целей;

- уровень дублирования и игнорирования функций - это соотношение общего количества подразделений к подразделениям, имеющим дублируемые и игнорируемые функции; 
- кадровый состав - это соотношение удельного веса должностей к общему числу сотрудников;

- соотношение количества административно-управляющего персонала к численному составу подчиненных (мастеров, рабочих);

- интенсивность взаимодействия внутренних связей между подразделениями, а также процесс их коммуникации с внешней средой.

Ожидаемым результатом процесса реструктуризации с последующим формированием показателей организационной эффективности предполагается повышение степени оперативности принимаемых решений за счет своевременности и быстрого реагирования к вызовам внешней среды.

\section{Заключение}

Внедряя предлагаемую концептуальную модель реструктуризации организационной структуры управления, спецавтохозяйства смогут достичь оперативности ПУР, избежать дублирования функций между отделами и улучшить между ними коммуникацию, а также актуализировать процесс инновации с помощью внедрения отдела информационных технологий как одного из базисного элемента организационной структуры.

По сравнению с существующей организационной структурой, предлагаемая модель является более динамичной и гибкой при переходе к другому типу структур. Реальность такова, что внешняя среда предприятий формирует вызовы в виде участия в национальных проектах, региональных и муниципальных программах и т. д. [19].

Для того чтобы учитывать данные вызовы и планомерно на них реагировать, предприятию необходимо определиться с типом организационной структуры, от которой зависит эффективность принимаемых управленческих решений и результативность всей работы предприятия.

Одним из таких видов является матричная организационная структура. В данном случае разработанная концептуальная модель является промежуточным звеном перехода от функциональной организационной структуры к проектно-матричной.

Вопрос о реструктуризации существующей структуры и переходе к проектноматричной стал актуальным для УМП «Спецавтохозяйство г. Томска» по причине того, что оно стало региональным оператором по вывозу твердых коммунальных отходов (ТКО) с территории Томска и Томского района в седьмой территориальной зоне [20]. Для того чтобы реализовать данную программу перед УМП «Спецавтохозяйство г. Томска» встала задача о проведении преобразований в существующей организационной структуре, добавив в нее проектно-матричные элементы.

\section{СПИСОК ЛИТЕРАТУРЫ}

1. 12 новых национальных проектов президента России. URL: http://www.gazetaprotestant.ru/2018/05/12novyx-nacionalnyx-proektov-prezidenta-rossii/ (дата обращения 17.04.2019).

2. Стоим в пробках: как вовремя попасть на работу. URL: https://www.tomsk.ru/news/view/138127 (дата обращения 15.04.2019).

3. Itzen P. Who is responsible in winter? Traffic accidents, the fight against hazardous weather and the role of law in a history of risks // Historical Social Research. - 2016. - № 1 (41). - P. 154-175.

4. Исследование талой воды (снега) как показатель загрязнения атмосферы урбанизированной среды / Н.Е. Соловьева, Е.А. Олькова, А.А. Алябьева, О.В. Краева // Молодой ученый. - 2015. - № 14. C. 668-672.

5. Обоснование применения модульной снегоплавильной установки для утилизации снега с территорий жилых домов и торговых центров / А.В. Шаруха, В.О. Довбыш, В.П. Шитый, С.В. Стрельбицкая // Современные проблемы науки и образования. - 2014. - № 6. - С. 354-361. 
6. Winter ecology of a subalpine grassland: effects of snow removal on soil respiration, microbial structure and function / K. Gavazova, J. Ingrischb, R. Hasibederb, Millsc R.T.E., A. Buttlerdef, G. Gleixnerg, J. Pumpanenh, M. Bahnb // Science of the Total Environment. - 2017. - V. 590-591. - P. 316-324.

7. Zakharov D., Magaril E., Rada E. Sustainability of the urban transport system under changes in weather and road conditions affecting vehicle operation // Sustainability. - 2018. - № 6 (10). - P. 1-17.

8. ОДМ 218.8.002-2010. Методические рекомендации по зимнему содержанию автомобильных дорог с использованием специализированной гидрометеорологической информации (для опытного применения). - М.: ИНФОРМАВТОДОР, 2010. - 52 с.

9. Снег на голову: как власти Томска «вывозят» проблему. URL: https://www.riatomsk.ru/ article/20170111/uborka-snega-tomsk-reshenie-problemi/ (дата обращения 15.04.2019).

10. Абакумов Р.Г., Унежева В.А., Страхова А.С. Анализ системных проблем жилищно-коммунального хозяйства города Белгорода и применение зарубежного опыта развития инновационной деятельности в системе жилищно-коммунального хозяйства // Вестник БГТУ им. В. Г. Шухова. - 2016. - № 6. С. 226-234.

11. Исследование влияния инфраструктуры городского хозяйства на региональное социальноэкономическое развитие / Е.В. Уфимцева, И.В. Волчкова, Ю.В. Подопригора, М.Н. Данилова, Н.Р. Шадейко, А.А. Селиверстов // Региональная экономика: теория и практика. - 2017. - Т. 15. № 2 (437). - С. 237-253.

12. Аспекты практической реализации алгоритма оценки комплексного развития инфраструктуры городского хозяйства / Н.Р. Шадейко, А.А. Селиверстов, Е.В. Уфимцева, И.В. Волчкова, М.Н. Данилова, Ю.В. Подопригора // Сибирская финансовая школа. - 2016. - № 6 (119). - С. 11-14.

13. Формирование системы показателей оценки комплексного развития городской инфраструктуры / Е.В. Уфимцева, И.В. Волчкова, М.Н. Данилова, Н.Р. Шадейко, Ю.В. Подопригора, А.А. Селиверстов // Вопросы управления. - 2016. - № 3 (21). - С. 81-91.

14. Jing Wang, Haotian Liu. Snow removal resource location and allocation optimization for urban road network recovery: a resilience perspective // Journal of Ambient Intelligence and Humanized Computing. 2019. - № 1 (10). - P. 395-408.

15. Дегтярева Н.А. Разработка управленческих решений. - Томск: Изд-во ТГУ. - 2018. - 100 с.

16. Григорьева Т.Е., Дегтярева Н.А. Процесс разработки и принятия управленческих решений в деятельности предприятий по зимнему содержанию дорог // Векторы благополучия: экономика и социум. 2018. - № 4 (31). - C. 266-275. URL: http://earchive.tpu.ru/handle/11683/52471 (дата обращения 20.05.2019).

17. Анализ и оценка основных фондов МУП «Спецавтохозяйство». URL: https://studbooks.net/1316560/ menedzhment/analiz_otsenka_osnovnyh_fondov_spetsavtohozyaystvo (дата обращения 15.04.2019).

18. Структура управления. URL: http://admnvrsk.ru/podrazdeleniya/munitsipalnye-byudzhetnye-i-unitarnyeuchrezhdeniya/mbu-spetsavtokhozyaystvo-administratsii-goroda/struktura-uprvleniya/ (дата обращения 15.04.2019).

19. Проекты благоустройства общественных пространств. URL: http://www.admin.tomsk.ru/pgs/a5i (дата обращения 02.05.2019).

20. В мэрии Томска решили разделить «Спецавтохозяйство» на два предприятия. URL: https://tomsk.mk.ru/social/2019/03/13/v-merii-tomska-reshili-razdelit-specavtokhozyaystvo-na-dvapredpriyatiya.html (дата обращения 25.04.2019).

Поступила 21.05.2019 2. 
UDC 005.591.4:338.47:656.135

\title{
DEVELOPMENT OF A CONCEPTUAL MODEL OF RESTRUCTURING THE ORGANIZATIONAL STRUCTURE OF SPECIAL MOTOR TRANSPORT SERVICE MANAGEMENT
}

\author{
Tatyana E. Grigoryeva1, \\ tanya_grig_1991@mail.ru \\ Natalia A. Degtyareva², \\ zxasqw@scalpnet.ru \\ ${ }^{1}$ Tomsk State University of Control Systems and Radioelectronics, \\ 40, Lenin avenue, Tomsk, 634050, Russia \\ ${ }^{2}$ Tomsk Branch of the Russian Presidential Academy \\ of National Economy and Public Administration, \\ 99, Lenin avenue, Tomsk, 634050, Russia
}

Tatyana E. Grigoryeva, postgraduate student, Tomsk State University of Control Systems and Radioelectronics.

Natalia A. Degtyareva, Cand. Sc., head of the Department of Humanities and Science Disciplines, Tomsk Branch of the Russian Presidential Academy of National Economy and Public Administration.

Various institutions and organizations, for example, housing and public utilities, management companies, the municipal unitary enterprise "Spetsavtokhokhyaystvo» and others that create comfortable urban environment for population, are involved in supporting the welfare of the city, its functioning and life support. The activity of the special motor transport services plays a special role in maintaining this environment. Their work is acquires significance in the period of heavy precipitation in the form of snow, which is accompanied by difficult movement of vehicles, including untimely snow, which negatively affects the ecological condition of the city. Analysis of the situation on roads shows that the processes of winter maintenance of roads require special efficiency in their organization and management. The object of the research is the generalized organizational structure of special motor transport service management, which corresponds to the linearfunctional type. There are a number of shortcomings with such a structure that lead to a loss of decisionmaking efficiency later, which is so important in the activities of special motor transport services, as they have close relationship with a dynamically changing environment. In this regard, the aim of the article is to develop a conceptual model of restructuring the existing management structure of special motor transport services. In developing such a model, methods of theoretical analysis with design elements were used. The proposed model is a functional organizational structure with elements of a linear one and it will allow the special motor transport services to achieve rapid management decision making, avoid duplication of functions between departments and improve communication between them. The advantages of the existing organizational structure remain and its disadvantages are minimized in the transition to the proposed structure. Moreover, the proposed model is dynamic and flexible when moving to a different type of structure. In the case of participation of special motor transport services in national, federal projects, regional and municipal programs, etc., it is necessary to add the project-matrix elements into organizational structure.

Key words: Winter maintenance, special motor transport services, management decision making, organizational structure and its restructuring, urban environment.

\section{REFERENCES}

1. 12 novykh natsionalnykh proektov prezidenta Rossii [12 new national projects of the President of Russia]. Available at: http://www.gazetaprotestant.ru/2018/05/12-novyx-nacionalnyx-proektov-prezidenta-rossii/ (accessed 17 April 2019). 
2. Stoim v probkakh: kak vovremya popast na rabotu [We are in traffic jams: how to get to work in time]. Available at: https://www.tomsk.ru/news/view/138127 (accessed 15 April 2019).

3. Itzen P. Who is responsible in winter? Traffic accidents, the fight against hazardous weather and the role of law in a history of risks. Historical Social Research, 2016, no. 1 (41), pp. 154-175.

4. Soloveva N.E., Olkova E.A., Alyabeva A.A., Kraeva O.V. Issledovanie taloy vody (snega) kak pokazatel zagryazneniya atmosfery urbanizirovannoy sredy [The study of melt water (snow) as an indicator of atmospheric pollution of the urbanized environment]. Molodoy ucheny, 2015, no. 14, pp. 668-672.

5. Sharukha A.V., Dovbysh V.O., Shity V.P., Strelbitskaya S.V. The rationale for the use of modular snow melting installation for the disposal of snow from the territories of residential buildings and shopping centers. Sovremennye problemy nauki i obrazovaniya, 2014, no. 6, pp. 354-361. In Rus.

6. Gavazova K., Ingrischb J., Hasibederb R., Millsc R.T.E., Buttlerdef A., Gleixnerg G., Pumpanenh J., Bahnb M. Winter ecology of a subalpine grassland: effects of snow removal on soil respiration, microbial structure and function. Science of the Total Environment, 2017, vol. 590-591, pp. 316-324.

7. Zakharov D., Magaril E., Rada E. Sustainability of the urban transport system under changes in weather and road conditions affecting vehicle operation. Sustainability, 2018, no. 6 (10), pp. 1-17.

8. ODM 218.8.002-2010. Metodicheskie rekomendatsii po zimnemu soderzhaniyu avtomobilnykh dorog $s$ ispolzovaniem spetsializirovannoy gidrometeorologicheskoy informatsii (dlya opytnogo primeneniya) [ODM 218.8.002-2010. Guidelines for winter maintenance of roads using specialized hydrometeorological information (for experimental use)]. Moscow, INFORMAVTODOR Publ., 2010. 52 p.

9. Sneg na golovu: kak vlasti Tomska "vyvozyat» problemu [Snow on the head: how the authorities of the Tomsk "take out" the problem]. Available at: https://www.riatomsk.ru/article/20170111/ uborka-snegatomsk-reshenie-problemi/ (accessed 15 April 2019).

10. Abakumov R.G., Unezheva V.A., Strakhova A.S. Analysis of the system problems of housing and communal services of the city of Belgorod and the application of foreign experience in the development of innovative activities in the system of housing and utilities. Vestnik BGTU im. V.G. Shukhova, 2016, no. 6, pp. 226-234. In Rus.

11. Ufimtseva E.V., Volchkova I.V., Podoprigora Yu.V., Danilova M.N., Shadeyko N.R., Seliverstov A.A. Study of the impact of urban infrastructure on regional socio-economic development. Regionalnaya ekonomika: teoriya i praktika, 2017, vol. 15, no. 2 (437), pp. 237-253. In Rus.

12. Shadeyko N.R., Seliverstov A.A., Ufimtseva E.V., Volchkova I.V., Danilova M.N., Podoprigora Yu.V. Aspekty prakticheskoy realizatsii algoritma otsenki kompleksnogo razvitiya infrastruktury gorodskogo khozyaystva [Aspects of the practical implementation of the algorithm for assessing the integrated development of urban infrastructure]. Sibirskaya finansovaya shkola, 2016, no. 6 (119), pp. 11-14.

13. Ufimtseva E.V., Volchkova I.V., Danilova M.N., Shadeyko N.R., Podoprigora Yu.V., Seliverstov A.A. Formirovanie sistemy pokazateley otsenki kompleksnogo razvitiya gorodskoy infrastruktury [Formation of a system of indicators for assessing the integrated development of urban infrastructure]. Voprosy upravleniya, 2016, no. 3 (21), pp. 81-91. In Rus.

14. Jing Wang, Haotian Liu. Snow removal resource location and allocation optimization for urban road network recovery: a resilience perspective. Journal of Ambient Intelligence and Humanized Computing, 2019, no. 1 (10), pp. 395-408.

15. Degtyareva N.A. Razrabotka upravlencheskikh resheniy [Development of management decisions]. Tomsk, Tomsk State University Publ., 2018. 100 p.

16. Grigoreva T.E., Degtyareva N.A. Development and management decisions making in activity of enterprises for winter maintenance of roads. Journal of Wellbeing Technologies, 2018, no. 4 (31), pp. 266-275. In Rus. Available at: http://earchive.tpu.ru/handle/11683/52471 (accessed 20 May 2019).

17. Analiz i otsenka osnovnykh fondov MUP «Spetsavtohozyaystvo» [Analysis and assessment of fixed assets of MUP «Spetsavtokhokhyaystvo»]. Available at: https://studbooks.net/1316560/menedzhment/analiz otsenka_osnovnyh_fondov_spetsavtohozyaystvo (accessed 15 April 2019).

18. Struktura upravleniya [Management structure]. Available at: http://admnvrsk.ru/podrazdeleniya/munitsipalnyebyudzhetnye-i-unitarnye-uchrezhdeniya/mbu-spetsavtokhozyaystvo-administratsii-goroda/strukturauprvleniya/ (accessed 15 April 2019).

19. Proekty blagoustroystva obshchestvennykh prostranstv [Public space improvement projects]. Available at: http://www.admin.tomsk.ru/pgs/a5i (accessed 2 May 2019).

20. V merii Tomska reshili razdelit «Spetsavtokhozyaystvo» na dva predpriyatiya [The Tomsk mayor's office decided to divide the «Spetsavtokhokhyaystvo» into two enterprises]. Available at: https://tomsk.mk.ru/ social/2019/03/13/vmerii-tomska-reshili-razdelit-specavtokhozyaystvo-na-dva-predpriyatiya.html (accessed 25 April 2019).

Received: 21 May 2019. 